\title{
„Bei einer sinnvollen Strukturanpassung der Medizinlandschaft ist der Patient der Hauptnutznießer jeder Teleradiologie“
}

Dr. Christian Rosenberg, Chefarzt der Klinik für Bildgebende Diagnostik und Interventionsradiologie, Evangelisches Waldkrankenhaus Spandau, erläutert im Interview, wieviel digitale Kommunikation in der Teleradiologie steckt und weshalb sie ein wichtiger Baustein für eine flächendeckende und qualitativ hochwertige medizinische Versorgung von Patienten sein kann. Rosenberg war zuvor leitender Oberarzt am Institut für Diagnostische Radiologie und Neuroradiologie der Universitätsmedizin Greifswald und in dieser Funktion aktiv eingebunden in das Netzwerk „Telemedizin in der Euroregion POMERANIA e. V.“

Herr Dr. Rosenberg, eines der Schwerpunktthemen des diesjährigen Deutschen Röntgenkongresses lautet Digitale Kommunikation in der Radiologie. Wie viel Kommunikation steckt in der Radiologie?

Eine Menge! Bilddaten und anhängender Befund zu einer ärztlich-radiologischen Leistung werden in digitaler Form transportiert, das ist Kommunikation. Die Inhalte dieses Pakets patientenspezifisch in einen medizinischen Kontext zu stellen, ist mindestens genauso wichtig. Diese Kommunikation - nennen wir sie Kontextkommunikation - findet gegenüber dem Patienten und/oder mitbehandelnden Kollegen statt. In der Sache unterscheidet sich hier das ambulante Setting, also ein Arztnetzwerk, nicht vom klinischen Setting, z. B. einer multidisziplinären Tumorkonferenz.

\section{Was genau versteht man unter Teleradio- logie?}

Im weiteren Sinne bedeutet Teleradiologie, dass Komponenten dieser radiologischen Leistung räumlich getrennt stattfinden, also Teile der Untersuchung bzw. Befundung, der Befundkommunikation oder der Kontextkommunikation. Die Mitbeurteilung von Bildern durch Kollegen einer anderen Fachdisziplin - anstelle einer direkten Kommunikation mit dem befundenden Radiologen - kann mit teleradiologischen
Mitteln erfolgen. Ebenso die Zweitbeurteilung durch einen fachkundigen Radiologen, z. B. im Rahmen einer orts- und zweckgebundenen Spezialfragestellung. Nichts Anderes findet statt, wenn im Rahmen einer standortübergreifenden interdisziplinären Videokonferenz sachbezogen radiologische Bilddaten auf einem geteilten Bildschirm aufgerufen und fachkundig beurteilt werden. Teleradiologie im engeren Sinne meint die Durchführung einer Untersuchung ohne körperliche Anwesenheit eines fachkundigen Radiologen am selben Ort. Diese Form der Teleradiologie ist nur als Ausnahme von der Regel erlaubt und für Verfahren mit Nutzung ionisierender Strahlen im Strahlenschutzgesetz geregelt. Ein an anderem Ort befindlicher fachkundiger Radiologe steht in Echtzeit und durch telekommunikative Mittel mit den Akteuren vor Ort in Verbindung, er trägt und behält die unmittelbare Untersuchungsverantwortung. Nachvollziehbarerweise gewinnt die Gewährleistung einer suffizienten Kontextkommunikation in diesem Setting eine besondere Bedeutung.

\section{Wann macht Teleradiologie überhaupt Sinn und für wen?}

Teleradiologie macht zweifellos Sinn, wenn durch sie eine unvermeidbare örtlich oder zeitlich begrenzte Unterversorgung von $\mathrm{Pa}$ tienten kompensiert werden kann. Diese medizinisch-radiologische Unterversorgung kann absolut sein - der Einsatz eines fachkundigen Radiologen vor Ort ist nicht möglich, oder sie kann die Versorgungsqualität betreffen - wenn z. B. in einem übergeordneten STROKE-Netzwerk die erforderliche Fachexpertise mit teleradiologischen Mitteln in die regionale Peripherie gebracht wird, um eine unnötige Verlegung des Patienten zu verhindern. Sie kann helfen, mehrere Standorte eines Versorgungszentrums zu vernetzen und dadurch einer übermäßigen Zentralisierung der Gesundheitsversorgung entgegenwirken. Teleradiologie ist, zumindest aus medizinischer Sicht, immer dann nicht sinnvoll, wenn Personaleinsparung im Rahmen von Rationalisierungsmaßnahmen, die Gewinnmaximierung Dritter oder Fehlplanung bei der

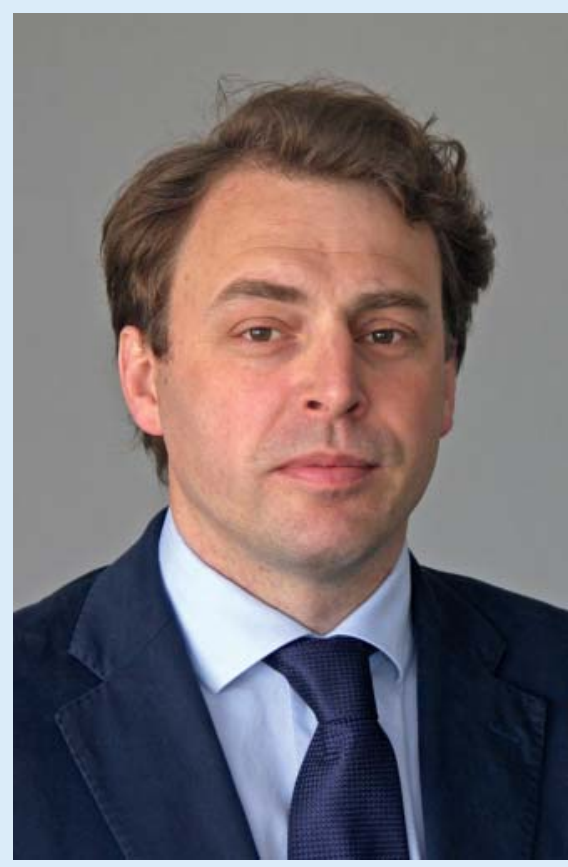

Dr. Christian Rosenberg

Nachwuchsausbildung den primären Impuls zu ihrer Implementierung darstellen.

Welchen konkreten Nutzen bietet die Teleradiologie also für den Patienten?

Bei einer sinnvollen Strukturanpassung der Medizinlandschaft ist der Patient der Hauptnutznießer jeder Teleradiologie; vielmehr sollte der unmittelbare Nutzen für den Patienten ein verpflichtender Maßstab für jede systematische Einführung von Teleradiologie sein. Der Vorteil für den Patienten kann in einer Anhebung der allgemeinen und individuellen Versorgungsqualität bestehen, sicher aber in einer Einsparung unnötiger Wege für den Patienten, seine persönlichen Daten und auch für die behandelnden Ärzte - das hat Potential zur nachhaltigen Kosteneinsparung. Als Bremse für eine versorgungspolitisch unsinnige übermäßige Zentralisierung von Gesundheitsversorgung kann Teleradiologie zum Erhalt einer heimatnahen Erreichbarkeit von Gesundheitszentren beitragen. 


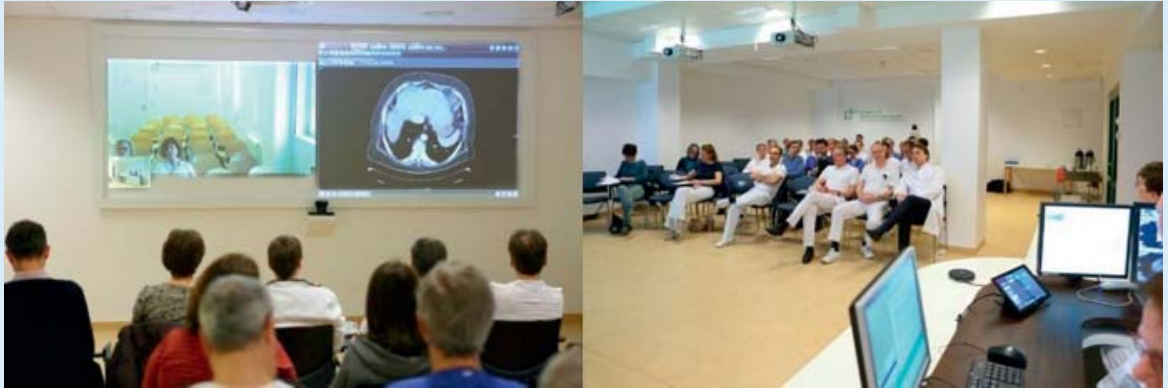

Eine standortübergreifende Tumorkonferenz im Evangelischen Waldkrankenhaus Spandau mit KollegInnen der Evangelischen Lungenklink Berlin. @ M. Tennert

Wie hat man sich den Prozess dahinter genau vorzustellen, z. B. im Rahmen der Notfallversorgung?

Die typische Konstellation ist ein kleineres Haus mit CT und Rettungsstelle. Ein radiologischer Nacht- oder Wochenenddienst wird nicht vorgehalten. Mit Hilfe eines vertraglich gebundenen Teleradiologen kann die Rettungsstelle 24/7 betrieben werden. Die technische Durchführung erfolgt durch eine fachkundige MTRA. Rechtfertigende Indikation, Untersuchungsverantwortung, Befundung und Befundkommunikation übernimmt der Teleradiologe.

Wie sieht die Arbeitsteilung aus und welche Qualifikationen sind im Einzelnen erforderlich?

Der diensthabende Arzt in der Notaufnahme spielt eine Rolle. Gemäß vertraglicher Bindung übernimmt er im Auftrag seines Arbeitgebers die Erstuntersuchung des zuzuführenden Patienten und teilt seine Informationen mit dem Teleradiologen. Sein Zuständigkeitsbereich für vitale und andere akute Notfälle wird auf die CT-Suite ausgedehnt. Er benötigt zertifizierte Grundkenntnisse im Strahlenschutz, je nach Bundesland zusätzlich eine Teilfachkunde in Teleradiologie. In der Regel übernimmt er die Untersuchungsaufklärung. Ob diese ausreicht oder ob beispielsweise eine Aufklärung durch den Teleradiologen per Videokonferenz erfolgen muss, ist juristisch umstritten - in einer echten Notfallsituation nachgeordnet. Nach Beauftragung durch den (derzeit voll fachkundigen) Teleradiologen führt die fachkundige MTRA die indizierte Untersuchung technisch durch und übermittelt den Bilddatensatz an den Teleradiologen. Dieser erstellt den schriftli- chen Befund und übermittelt diesen in geeigneter Form.

Ist Teleradiologie nicht technisch sehr aufwändig und mitunter störanfällig? Schließlich werden ja große Datenmengen bewegt und dies insbesondere im ländlichen Raum mit einem tendenziell anfälligeren Leitungsnetz?

Die technischen Anforderungen lassen sich organisieren, die Zuständigkeiten müssen klar geregelt sein. Ein Ausfall der Technik ist dennoch nie vollständig ausgeschlossen.

Wie wird verhindert, dass patientenbezogene Daten nicht in die Hände Dritter gelangen?

Die Anforderungen des Datenschutzes sind technisch lösbar. Jede Kooperation basiert auf einem vertraglichen Fundament, das vorher durch die Datenschützer abgenommen wird. Teleradiologie kann je nach Organisationsstruktur die Patientenautonomie stärken, dem Patienten nachhaltig die Hoheit über seine eigenen Daten sichern.

Was passiert eigentlich, wenn teleradiologische Leistungen nicht abgerufen werden können, z. B. aufgrund technischer Probleme. Ist dann gewährleistet, dass ein Radiologe trotzdem schnell verfügbar ist?

Es gibt im Strahlenschutzgesetz das sogenannte Regionalprinzip, das vorschreibt, dass der Teleradiologe in angemessener Zeit - nach Gesetzesauslegung innerhalb von 45 Minuten - den Untersuchungsort erreichen kann. Zukünftig soll der Teleradiologe zusätzlich eine dienstliche Verbindung mit der versorgten Einrichtung doku- mentieren, z. B. durch eine regelmäßige Vorort-Präsenz.

Wie viele teleradiologische Dienstleister gibt es in Deutschland bzw. wer bietet überhaupt solche Leistungen an?

In einer Vielzahl von Spielarten gibt es landesweit regionale Versorgungsstrukturen, in denen größere medizinische Versorgungseinheiten 24/7-Versorgung vorhalten und kleinere Einheiten außerhalb regulärer Dienstzeiten teleradiologisch mitversorgen. Dem jeweiligen Bedarf angepasst, erfolgt diese Kooperation zwischen Kliniken unterschiedlicher oder gleicher Trägerschaft. Mit fallender Bevölkerungsdichte steigt dabei die zentripetale Bedeutung des Leistungsanbieters. Darüber hinaus gibt es eine Anzahl von Anbietern, häufig überregional als Privatunternehmen oder MVZ organisiert, die sich auf das Angebot teleradiologischer Leistung spezialisiert haben.

Teleradiologie bedeutet ja neben der radiologischen Befundung eines Patienten durch einen Radiologen, der nicht vor Ort ist, auch die digitale Übertragung von Röntgen- und CT-Aufnahmen und den fachlichen Austausch zur Beurteilung der Befunde. Die sogenannte „telekonsiliarische Befundbeurteilung“ von Röntgenaufnahmen ist durch das E-Health-Gesetz auch Teil der vertragsärztlichen Versorgung. Haben sich Telekonsile in der Versorgungspraxis bereits etablieren können?

Ja, die Kontextkommunikation ist sehr wichtig. Jeder Radiologe nimmt dieses Element seiner ärztlichen Tätigkeit sehr ernst. Gerade jüngere Kollegen können sich hierdurch nachhaltig profilieren. Die fragestellungsbezogene Zweitbeurteilung von Fremdaufnahmen ist schon immer Bestandteil der Praxis. Die entsprechende Würdigung im vertragsärztlichen Tarifsystem ist ein Schritt in die richtige Richtung. Dennoch sind Fragen der Abrechenbarkeit weiterhin ungeklärt. Ungeklärt ist auch eine analoge Abrechnung im Rahmen der sektorenübergreifenden Versorgung, z. B. für ambulante Patienten in einer klinischen Tumorkonferenz. Wie ist bei der ebenfalls aus dem E-Health-Gesetz abgeleiteten „Zweitmeinung“vor wichtigen Eingriffen 
die fachkundige radiologische Mitbeurteilung gesichert? Wie ist die Einbindung klinischer Spezialisten geregelt?

Ist die Teleradiologie in ihren unterschiedlichen Ausprägungen mit Blick auf die demografische Entwicklung und den Fachärztemangel in strukturschwachen Regionen alternativlos?

Sicherlich nicht. Ist Mangel oder Strukturschwäche Ursache für die Implementierung mithin sogar einer zeitlich begrenzten Primärversorgung durch Teleradiologie, so sollten Politik und Fachverbände im Patienteninteresse geeignete Maßnahmen ergreifen, um diesem Mangel entgegenzuwirken. Wo Teleradiologie zu einer Verbesserung der Versorgungsqualität führt, wo sie sinnvolle Synergien in der regionalen Gesundheitsversorgung trägt, ist sie alternativlos.

\section{Veranstaltungstipps}

\section{Mai 2018}

Highlight 101: Teleradiologie nach RöV oder geht doch noch mehr?

Vorsitz/Moderation: Prof. Dr. Gerald Weisser (Mannheim), Prof. Dr. Dr. Reinhard Loose (Nürnberg)

14:45 bis 16:15 Uhr, Raum Rieder

\section{MTRA 4: Digitale Kommunikation}

Vorsitz/Moderation: Brigitte Olbrich (Greifswald)

17:00 bis 18:00 Uhr; Raum Kundt

\section{Mai 2018}

Highlight 204: Eröffnung der 99. Deutschen Röntgenkongresses

Vorsitz/Moderation: Prof. Dr. Peter Huppert (Darmstadt), Prof. Dr. Stefan Schönberg
(Mannheim)

11:00 bis 12:30 Uhr, Raum Röntgen

\section{Mai 2018}

Highlight 303: Röntgen-Vorlesung

Vorsitz/Moderation: Prof. Dr. Stefan Schönberg (Mannheim), Prof. Dr. Peter Huppert (Darmstadt)

11:00 bis 12:30 Uhr, Raum Röntgen

SP 313: Radiologie und IT/Neuro IX - Teleradiologische Netzwerke in der Neuroradiologie

Vorsitz/Moderation: Dipl.-Phys. Roland Simmler (Zürich), Prof. Dr. Gerald Weisser (Mannheim)

17:00 bis 18:30 Uhr, Raum Rieder 